\title{
Origen social y procesos escolares en Paraguay. Factores asociados al rendimiento académico en lectura y matemática de estudiantes del tercer grado
}

\section{Social origin and school processes in Paraguay. Factors associated to the academic performance in reading and mathematics of third grade students}

\section{Ana Teresa Recalde}

Instituto Nacional de Evaluación Educativa, Paraguay

\section{Resumen}

Este estudio de enfoque cuantitativo y alcance explicativo realizado con información proveída por el Laboratorio Latinoamericano de Evaluación de la Calidad Educativa (LLECE)-UNESCO pretende establecer y comparar los factores de contexto y proceso asociados al rendimiento en el Tercer Estudio Regional Comparativo y Explicativo (TERCE) en las áreas de lectura y matemática de estudiantes del tercer grado, en instituciones escolares de la República del Paraguay durante el año 2013.

Al efecto se analizaron 2759 exámenes junto a la información de cuestionarios auto-administrados al estudiante, su familia, el docente del aula y el director del establecimiento, para 200 instituciones educativas.

Para la estimación de los factores asociados, se recurrió a un modelo lineal jerárquico que estableció como factores de mayor asociación con el rendimiento en las áreas en cuestión al capital cultural del estudiante y los recursos materiales de la escuela, asi como, en menor medida, el entorno del aula. Además, en el área de lectura se asocian significativamente la gestión del director -con efecto negativo-, el origen social del estudiante y el hábitat de la escuela. Asimismo, resultaron significativas $(p<0,01)$ las incidencias diferenciales de las categorías (alto/bajo) de los factores contextuales (origen social, capital cultural, entorno del aula, hábitat, dependencia y recursos materiales) entre los rendimientos de las pruebas.

Palabras clave: factores asociados, desigualdad educativa, rendimiento académico, reproducción, eficacia escolar.

\begin{abstract}
This is a quantitative study of explanatory scope realized with information provided by The Latin American Laboratory of Evaluation of the Educational Quality (LLECE)-UNESCO with the purpose of establishing and comparing context and process factors associated with performance in the TERCE assessment of Reading and Mathematics learning by 3rd graders in school institutions of the Republic of Paraguay during the year 2013.

For this purpose, 2759 exams were analyzed together with the information of selfadministered questionnaires to the student, his family, the classroom teacher and the director of 200 educational establishments.

For the estimation of the associated factors a hierarchical linear model was used that established as factors of greater association, with the performance in the Reading and Mathematics assessments, the Cultural Capital of the student, the School Resource Materials and to a lesser extent the Class Climate. In addition, reading is significantly
\end{abstract}


associated with the director's management -with negative effect-, the social origin of the student and the school's habitat. Likewise, the differential incidences of the categories (high / low) of the contextual factors (social origin, cultural capital, classroom environment, habitat, dependency and material resources) among the test performances were significant $(p<0.01)$.

Keywords: associated factors, educational inequality, academic performance, reproduction, school effectiveness

\section{INTRODUCCIÓN}

La educación es tarea asumida por los Estados, para la cual se instrumentan legislaciones, se estructura un sistema, se asignan recursos y se delinean políticas educativas, reflejadas en la implementación de programas académicos en las escuelas, y estas se traducen en objetivos de aprendizaje en las aulas de clase. El Estado paraguayo define en el Plan Nacional de Educación 2024 la política educativa como un marco referencial complejo centrado en lo pedagógico, estableciendo a través de su órgano rector -el Ministerio de Educación y Ciencias (MEC)- las directivas conceptuales, técnicas, éticas y políticas del proceso educativo, definiéndolo como el "quehacer dinámico que se da en las relaciones educativas cotidianas que hacen a la formación de las personas como seres originales y protagonistas activos en la construcción del contexto sociocultural del Paraguay contemporáneo" (MEC, 2011, pp. 46-48).

De igual modo, el Plan Nacional de Educación 2024 concibe un sistema educativo inclusivo, con equidad y de calidad, como atributos destacados y al que adhiere la planificación según lo expresado en la Agenda Educativa 2013-2018 bajo la máxima "Todas las personas, independientemente del lugar de donde provienen y del nivel socioeconómico al que pertenecen, nacen con similares potencialidades de talentos". (MEC, 2014, pp. 22-23). A fin de operacionalizar los aspectos mencionados, funcionalmente, la educación se desdobla a través de la enseñanza como proceso y el aprendizaje como producto. En el proceso de enseñanza es necesario considerar ciertas condiciones de base del estudiante y su contexto, para su estudio se denominan factores. Como producto, el aprendizaje-del estudiante-se traduce en un resultado susceptible de valoración a través de su rendimiento académico, una forma de evaluarlo por ejemplo sería a través pruebas estandarizadas.

En las investigaciones que abordan factores asociados al rendimiento académico de los estudiantes, se consideran ambas dimensiones, proceso y producto, así como el contexto donde estas se encuentran, la escuela, ante eso cabe la pregunta ¿cuánto influye la escuela para cambiar la situación de un estudiante en todas sus dimensiones?, para dar respuesta se requiere el estudio de los factores implicados. 


\section{PLANTEAMIENTO DEL PROBLEMA}

El estudio de los factores asociados al rendimiento académico de los estudiantes puede dividirse al menos en dos vertientes. Una, que ha puesto el peso en el estudiante y sus características; otra, en la escuela y sus procesos. Actualmente, tomando siempre como eje los resultados de las evaluaciones del rendimiento, se busca lograr una comprensión más ajustada de la realidad y las variables que la componen.

Históricamente, el informe Coleman marca un hito en el estudio de factores asociados al aprendizaje, mostrando la disponibilidad de oportunidades educativas en las escuelas públicas, los datos destacaban la gran desigualdad y segregación de los estudiantes y profesores negros, así como señalaba que el alumno medio correspondiente a las minorías realiza menos y es más afectado por la calidad de su escuela que el alumno "blanco" medio. (Coleman et al., 1966, pp. 3-33).

En Francia hacia 1965, la sociología hace su aporte al estudio de la educación desde el constructivismo estructuralista de Bourdieu y Passeron. Partiendo de la premisa de que toda cultura es arbitraria y su definición es siempre social, estudia el campo cultural estableciendo que la enseñanza sirve de modo específico a las estructuras sociales al reproducir las relaciones de clase como resultado de una acción pedagógica. La acción pedagógica impone significaciones como si fueran legítimas al través de una autoridad reconocida y es una función específicamente social de la escuela. Los sujetos reciben un capital cultural en primera instancia de la familia y luego de la escuela además de una serie de posturas con respecto a la cultura. La escuela es un agente de reproducción y legitimación de la diferenciación social, asegurando su interiorización y persuadiendo que ésta no es social, sino que hace parte de un "orden natural" (Bourdieu y Passeron, 1996)

En Latinoamérica, las investigaciones de factores asociados se han centrado en la eficacia escolar, estas datan de finales de 1970: Martínez-Garrido (2011) hace un recuento de lo que denomina "veinticinco investigaciones claves sobre eficacia escolar", organizando los estudios según su foco: i) aquellas que estudian los factores de la escuela y sus efectos en el desarrollo académico de los estudiantes, ii) las que abordan la enseñanza eficaz, identificando aspectos del proceso de esenañzan, iii) las que estudian la eficacia educativa que buscan integrar los conceptos de eficacia escolar y enseñanza escolar (Martínez-Garrido, 2011, p. 3). 
En general, la investigación educativa mundial tiene por objetivo estudiar la estratificación social de los aprendizajes, los efectos de la escuela y los efectos de las instituciones. Por otra parte, en la década de 1990, América latina inicia estudios sobre la desigualdad de aprendizajes que desplazan a los enfocados en la desigualdad de acceso a la educación obligatoria. Es entonces cuando convergen el enfoque sociológico de la teoría de la reproducción cultural de Bourdieu y Passeron con los estudios sobre la eficacia escolar. A modo de producto, en las comparaciones internacionales, se sustenta la tesis de que en los países desarrollados la escuela tiene poco o ningún efecto, mientras que en los del tercer mundo, su importancia relativa es mucho mayor.

Cervini (2002) se sitúa en ese marco conceptual y se "propone determinar el nivel de (in)equidad educativa existente en Argentina y la importancia relativa de los factores escolares institucionales (escuelas) y regionales (provincias) en la distribución del nivel de aprendizaje en matemática y lengua de la educación básica" ( $p$. 448). Constata que las mediciones de capital económico y cultural son predictores significativos de los puntajes en las pruebas de matemática y lengua, principalmente en su forma contextual (segmentación social del sistema educativo), aunque estos factores no explican los puntajes medios de las escuelas. Esto es, las características de la escuela o de la práctica pedagógica también serían factores significativos del nivel y distribución de los a prendizajes escolares en Argentina (Cervini, 2002, p. 445).

El estudio de Fernández (2004) plantea una relación entre la distribución desigual del aprendizaje cognitivo en estudiantes con enseñanza primaria (básica) concluida en Argentina, Chile, México y Uruguay y la estructura de clases así como las características organizacionales de las escuelas. Al efecto, analiza con modelos jerárquicos lineales las "pruebas nacionales del periodo 1999-2001, con la excepción de Chile"1 (Fernández, 2004, p. 31), recurriendo a la teoría de la reproducción cultural y a la teoría elección racional para comparar países, así como a diferentes teorías para el análisis organizacional de la escuela y mantiene en la discusión algunos aspectos de economía política como indicadores del bienestar social que se consideran de regulación externa respecto al funcionamiento de las escuelas. Entre sus hallazgos sobresale que en los 4 países el índice de capital familiar calculado es la variable

\footnotetext{
${ }^{1}$ Chile. Los datos provienen del Third International Mathematics and Science Study (TIMS, 1999). Argentina. Los datos provienen del Operativo Nacional de Evaluación (ONE, 1999). México. Los datos provienen de la aplicación de Estándares Nacionales en Español y Matemática (EN, 2001).

Uruguay. Los datos provienen de la Evaluación Nacional de Aprendizajes (UMRE, 1999).
} 
más determinante del aprendizaje, luego el trabajo infantil y un efecto directo "de los planes que los escolares empiezan a trazarse para su futuro" (Fernández, 2004, p. 534).

Dos hallazgos derivados son notables: en primer término, que cuanto más equitativa es la distribución del nivel de bienestar entre los habitantes, menor es el peso de la escuela en los aprendizajes, abriendo el debate sobre la tesis de que la estructuración de la desigualdad de aprendizajes se entiende en el marco del régimen de bienestar social. En segundo lugar, que la magnitud del "efecto escuela" hallado en un país, solamente informa que hay una fuerte limitación para mejorar la igualdad de aprendizajes. Ambos, llevan a objetar la idea predominante en ciertos países de que la política educativa sea responsable para disminuir la pobreza y mejorar la distribución del bienestar (Fernández, 2004, pp. 537-545).

Enfocando el tema a los estudiantes paraguayos y su participación en la evaluación del Tercer Estudio Regional Comparativo y Explicativo (TERCE), además de pertenecer al grupo de países con media significativamente menor al valor regional en todas las áreas evaluadas, Paraguay presentó alto grado de desigualdad (o variabilidad) en el desempeño de lectura en tercer grado, con brecha de 254 puntos. En mayor proporción estos estudiantes se ubican en el nivel I $\left(57,4 \%\right.$ ) y el 50,3\% del $6^{\circ}$ en el II (UNESCO, 2015, pp. 23-33). En matemática hubo mayor distribución en el nivel I con valores del $66,5 \%$ en tercer grado y $69,3 \%$ en sexto grado (UNESCO, 2015, pp. 51-62).

Más allá de los resultados, sistemáticamente bajos, de los estudiantes paraguayos en evaluaciones estandarizadas es poco lo que se discute para comprender funcionalmente los resultados. En ese sentido, Ortiz propone una mirada estructural del asunto: "La estructura social paraguaya está basada en desigualdades pronunciadas; el sistema educativo fue objeto de una reforma institucional, los diagnósticos abordaron tangencialmente las condiciones sociales; se hace necesario examinar esas condiciones de modo a establecer los alcances y límites del proyecto democrático y de la reforma educativa" (Ortiz, 2012, pp. 65-66). Las necesidades mencionadas, son las que se buscan abordar en esta investigación. 


\section{MARCO TEÓRICO}

El modelo de análisis propuesto por Bourdieu y Passeron desarrolla una relación lógica y coherente entre las relaciones sociales, es así, que la escuela y la cultura establecen los mecanismos de actuación de los condicionamientos sociales y su internalización en docentes y estudiantes. Al efecto, tratan la acción pedagógica como una relación de comunicación para medir su rendimiento en función de las características sociales y escolares de los estudiantes. Los resultados muestran que las prácticas culturales verbales y no verbales reflejan una sociedad condicionada - pero no determinada- por estructuras de clase en lo externo y hábitus en lo interno. La interpretación de los resultados -devenida en teoría- expone al sistema escolar como reproductor de la estructura social y de la cultura.

Según Bourdieu, la sociedad es concebida como un "campo de fuerzas" donde tienen lugar luchas entre agentes que "se enfrentan, con medios y fines diferenciados según su posición en la estructura, contribuyendo de este modo a conservar o a transformar su estructura" (Bourdieu, 1997, p. 49). Es así, que la organización de la sociedad opera como un espacio social caracterizado por posiciones distintas y coexistentes, diferentes las unas de las otras, definidas por relaciones de proximidad o distanciamiento y relaciones de orden jerárquico. En el espacio social, los agentes o los grupos se distribuyen en función de su posición en las distribuciones estadísticas según dos principios de diferenciación: el capital económico y el capital cultural (Bourdieu y Passeron, 2005, p. 30)

El capital cultural es un concepto que se refiere tanto a "los diversos componentes de una determinada cultura (sistema simbólico) como sus principales manifestaciones o productos" (Cervini, 2002, p. 458). Puede existir bajo tres formas o estados: i) incorporado, es decir, bajo la forma de disposiciones duraderas del organismo; ii) objetivado, bajo la forma de bienes culturales; iii) institucionalizado que es una forma particular de objetivación porque confiere al capital cultural propiedades originales tal como el título escolar.

En Latinoamérica, como ya se mencionó, la investigación sobre eficacia escolar se inicia en la década de 1970 con la característica de una orientación práctica, es decir, más centrada en la determinación de los factores asociados que ayudan a optimizar los niveles de calidad y equidad que en estimar la magnitud y las propiedades científicas de los efectos escolares. Las obras se enfocan tanto en el movimiento teórico-práctico de efectividad escolar como en la productividad escolar y se pueden 
agrupar en cuatro áreas: i) La eficacia escolar, ii) La relación entre determinados factores y el rendimiento, iii) Las evaluaciones de programas de mejora y iv) Los estudios etnográficos sobre la escuela (Murillo, 2003, pp. 26-27).

En el estado de la cuestión en Paraguay, Misiego realiza estudios sobre la temática abordada, manifestando que la infancia es un concepto relacionado con su clase social. Un niño de estrato social pobre rural carece de protección, sufre privaciones y su desarrollo cerebral insuficiente determina su salud, aprendizaje y conducta para toda la vida, "la pobreza material, social y psicosocial de su familia puede generarle menor disposición para aprender y escasa motivación para ir a la escuela con el consecuente bajo rendimiento, deserción y/o conductas de riesgo". (Misiego, 2014, pp. 5-7). Baird y Elías, en sus investigaciones concluyen que "la explicación del bajo rendimiento a nivel nacional, debe comenzar con el examen de las condiciones en el hogar, la familia, y otros aspectos del entorno en que se desarrollan los niños y niñas" (2014, p. 31)

Respecto a los hallazgos del TERCE, los estudiantes paraguayos se encuentran en situación de rezago en cuanto a su desempeño académico, en lectura más de mitad de los estudiantes de tercer grado $(57,4 \%)$ se encuentran en el nivel 1 , siendo apenas capaces de "localizar información explícita o repetida literalmente, extraer conclusiones a partir de ideas evidentes, inferir significados conocidos y reconocer tipos de textos breves". (UNESCO, 2015, pp. 24-25)

En matemática también la mayor cantidad de estudiantes se ubica en el nivel 1 $(66,5 \%)$, de esta manera la mayoría de ellos son capaces de "ordenar números naturales, comparar cantidades, identificar figuras geométricas básicas y elementos faltantes en secuencias simples, leer datos explícitos" (UNESCO 2015, p. 50). Con estas habilidades culminan los estudiantes paraguayos el primer ciclo de la educación primaria, con las consabidas consecuencias que el escaso repertorio de estas tiene en la trayectoria académica de un estudiante.

\section{MARCO METODOLÓGICO}

\subsection{Tipo de investigación, periodo y procedimientos}

Esta investigación contempla los factores de contexto y proceso que se relacionan con las puntuaciones en las pruebas TERCE logradas por alumnos del tercer grado de la educación escolar básica en Paraguay, de fuente secundaria, pues, se efectuó 
con información relevada por la UNESCO. Según el periodo y la secuencia, fue transversal. Por el análisis y alcance es de enfoque cuantitativo de alcance explicativo. Las evaluaciones del TERCE se aplicaron en Paraguay durante el año 2013.

Para la generación de los factores propuestos se utilizó el proceso de escalado a través del modelo de Teoría de Respuesta al Ítem, de tipo crédito parcial atendiendo la estructura politómica de las opciones de respuesta de los cuestionarios de los factores. En dicho proceso se tomaron los parámetros de confiabilidad, coeficiente alfa de Cronbach para la consistencia interna, relación de varianzas (unidimensionalidad) y separación para la aprobación de los factores. Se toman como parámetros ideales y los criterios de aceptación los valores expuestos en la Tabla 1.

TABLA 1

Parámetros estadísticos para la generación de índices

\begin{tabular}{lc}
\hline \multicolumn{1}{c}{ Parámetro } & Valor para la aprobación \\
\hline Confiabilidad & Mayor que 0,60 \\
Consistencia Interna & Mayor que 0,60 \\
Relación de varianzas & Mayor que 4,00 \\
Separación & Mayor que 4,00
\end{tabular}

Fuente: Adaptado de Bogoya et al (2014), p. 511

Asimismo, los indicadores considerados para admitir los ítems (preguntas) y su inclusión en los índices fueron la dificultad, error, correlación ítem-prueba, ajuste próximo y lejano, así como la discriminación, los valores ideales y criterios se exponen en la tabla 2. Finalmente, las variables se agruparon en torno a factores a partir del comportamiento empírico de las mismas según su grado de correlación con el puntaje en las pruebas y el soporte teórico propuesto.

TABLA 2

Valores ideales y criterios para admitir ítems

\begin{tabular}{lcc}
\hline \multicolumn{1}{c}{ Parámetro } & Valor ideal & Valor para la aprobación \\
\hline Dificultad & Entre -3 y 3 logit & Entre -3 y 3 logit \\
Error de la estimación & Menor que 0,01 & Menor que 0,18 \\
Ajuste próximo & 1,00 & Mayor que 0,60 y menor que 1,40 \\
Ajuste lejano & 1,00 & Mayor que 0,60 y menor que 1,40 \\
Discriminación & 1,00 & Mayor que 0,20 y menor que 1,80 \\
Correlación ítem-prueba & Entre 0,10 y 0,60 & Entre 0,10 y 0,60 \\
\hline
\end{tabular}

Fuente: Adaptado de Bogoya et al (2014), p. 510. 
Una vez generados los índices de los factores se efectuó un análisis multinivel a fin de identificar la magnitud de las diferencias del desempeño en matemática y lectura, en relación al origen social (factores extraescolares) de los estudiantes paraguayos de tercer grado y los procesos escolares (factores escolares).

Respecto al análisis multinivel, la metodología de análisis utilizada ha sido el modelo lineal jerárquico de dos niveles que considera pesos muestrales y valores plausibles. La misma tiene por objetivo modelar estadísticamente la influencia del contexto sobre las actitudes o comportamientos del individuo. En ciencias sociales permite estimar el efecto de las variables de la estructura social y económica sobre el individuo.

Las herramientas informáticas empleadas fueron los softwares Excel y SPSS (V.22) para la organización de la base de datos, para la ejecución de los comandos el Winsteps Rasch (V. 3.72.3), para el análisis multinivel se utilizó el software HLM (V.6.0) que trata modelos lineales jerárquicos a fin de obtener los coeficientes de los factores de cada uno de los niveles.

\subsection{Muestra}

TERCE, tuvo dos poblaciones objetivo, estudiantes de tercer y sexto grados, bajo los criterios de selección de pertenecer al sistema educativo formal y aptos para responder las pruebas disciplinares planteadas (lectura, matemática, ciencias, escritura), para el efecto se trabajó con una muestra de escuelas pertenecientes a la población definida, a través de un muestreo probabilístico bi-etápico, primero estratificado y después por conglomerados, "en la primera etapa se seleccionaron escuelas con probabilidad de selección proporcional al tamaño, y en la segunda etapa se seleccionó aleatoriamente un aula y se aplicó a todos los estudiantes pertenecientes a la misma". (UNESCO, 2016, pp. 200-206)

Para esta investigación la muestra fue intencional, pues se compone de las puntuaciones en cada una de las evaluaciones de lectura y matemática obtenidas por $n=3142$ estudiantes paraguayos de tercer grado que cumplieron los criterios de inclusión establecidos para las pruebas del TERCE. Sobre el total de 200 instituciones, 140 son del sector oficial, 31 subvencionadas y 29 privadas. En área urbana se ubican 102 y en zona rural 98. Respecto al área y sector, 2043 alumnos estudian en el área urbana y 1099 en el área rural, 2019 en escuelas del sector público, 613 en subvencionadas y 510 en instituciones privadas. 
Al calcular los índices de los factores se modificó el tamaño de la muestra en N=2759 exámenes: 1541 de lectura y 1218 de matemática, cuyas puntuaciones fueron objeto del análisis, por pérdida de respuestas para calcular los índices.

\subsection{Instrumentos de indagación empírica}

Los cuestionarios de contexto fueron desarrollados tomando en consideración el marco teórico del estudio. El TERCE cuenta con cuestionarios para estudiantes, familias, docentes y directores. La información consultada mediante estos instrumentos hizo posible efectuar análisis de factores asociados (UNESCO, 2013)².

\subsection{Justificación de la selección de las variables}

Esta investigación consideró los factores de contexto y proceso que se relacionan con las puntuaciones en las pruebas TERCE logradas por estudiantes del tercer grado. Al efecto, se seleccionaron elementos -preguntas- existentes en alguno de los cuestionarios de factores asociados para conformar una categoría de análisis. Dichos elementos son factibles de agruparse bajo una dimensión teóricamente coherente, también deben satisfacer la condición estadística de correlacionar significativamente con los puntajes de las disciplinas evaluadas. Ambas condiciones no son necesariamente simultáneas; si no correlacionan, prevalece la elección por ser parte de una variable latente. Esto puede considerarse la "parte empírica" que justifica la elección.

\footnotetext{
${ }^{2}$ Disponible en: http://www.unesco.org/new/es/santiago/terce/documents/
} 


\section{TABLA 3}

\section{Categorías y dimensiones de análisis del Nivel 1 Estudiante}

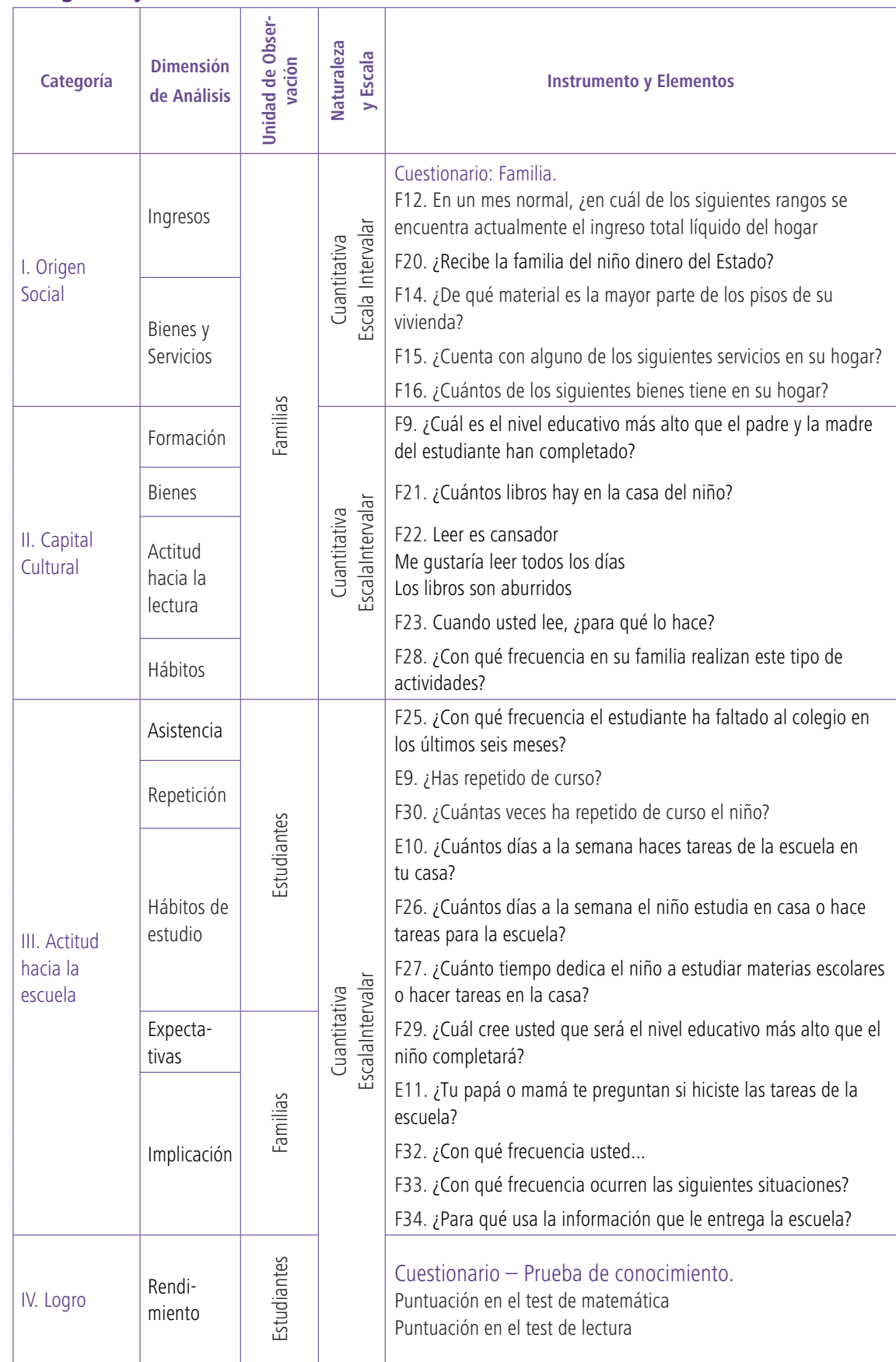




\section{TABLA 4}

\section{Categorías y dimensiones de análisis Nivel 2 Escuela}

\begin{tabular}{|c|c|c|c|c|}
\hline $\begin{array}{c}\text { Categoría } \\
\text { - Aula }\end{array}$ & $\begin{array}{l}\text { Dimensión } \\
\text { de Análisis }\end{array}$ & 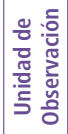 & 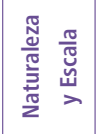 & Instrumento y Elemento \\
\hline $\begin{array}{l}\text { I. Re- } \\
\text { cursos } \\
\text { didácti- } \\
\text { cos }\end{array}$ & $\begin{array}{l}\text { Disponibi- } \\
\text { lidad }\end{array}$ & 总 & 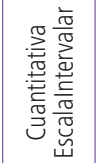 & $\begin{array}{l}\text { Cuestionario: Estudiante } \\
\text { E6. ¿Tienes texto de lenguaje para usar en clase? } \\
\text { ¿Tienes texto de matemática para usar en clase? } \\
\text { ¿Tienes cuadernos o libretas para tomar notas en clase? } \\
\text { En tu sala, ¿hay un estante o caja con libros para que ustedes lean? }\end{array}$ \\
\hline \multirow[b]{2}{*}{ II. Clima } & \multirow[b]{2}{*}{$\begin{array}{l}\text { Clima de la } \\
\text { sala }\end{array}$} & 号 & \multirow{2}{*}{ 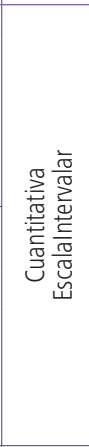 } & $\begin{array}{l}\text { E7. Durante tus clases... ¿Hay ruido y desorden en tu sala? } \\
\text { ¿Hay burlas entre compañeros? } \\
\text { ¿Los profesores se molestan con ustedes? } \\
\text { ¿Los alumnos nos quedamos sin nada que hacer? } \\
\text { ¿Los profesores tienen que esperar largo rato a que los alumnos nos } \\
\text { tranquilicemos? }\end{array}$ \\
\hline & & 苋 & & $\begin{array}{l}\text { Cuestionario: Profesor } \\
\text { P25. Puedo realizar mis clases sin interrupciones } \\
\text { Cuando estoy explicando algo, los estudiantes prestan atención } \\
\text { Disfruto mucho haciendo clases en este curso } \\
\text { Los estudiantes son agresivos entre sí } \\
\text { Los estudiantes suelen ayudar a los que les cuesta más } \\
\text { Los estudiantes muestran respeto por sus compañeros }\end{array}$ \\
\hline $\begin{array}{l}\text { Categoría } \\
\text { - Escuela }\end{array}$ & $\begin{array}{l}\text { Dimensión } \\
\text { de Análisis }\end{array}$ & 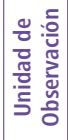 & 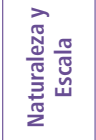 & Instrumento y Elemento \\
\hline I. Hábitat & Zona & 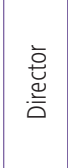 & 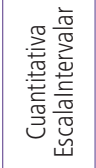 & $\begin{array}{l}\text { Cuestionario: Director } \\
\text { D11. Su escuela se encuentra en una localidad de: } 2000 \text { habitantes o } \\
\text { menos... } \\
\text { D12. ¿Cómo caracterizaría usted la zona donde está ubicada su escuela? }\end{array}$ \\
\hline $\begin{array}{l}\text { II. Re- } \\
\text { cursos } \\
\text { materia- } \\
\text { les }\end{array}$ & $\begin{array}{l}\text { Disponibi- } \\
\text { lidad }\end{array}$ & 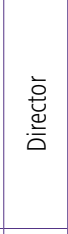 & 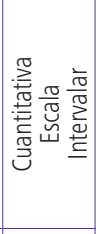 & $\begin{array}{l}\text { D14. ¿Con qué instalaciones cuenta la escuela? } \\
\text { D15. ¿Cuántos computadores hay en la escuela para uso de los } \\
\text { estudiantes? } \\
\text { D16. En cuanto al equipamiento de las aulas... } \\
\text { D17. ¿Con cuáles de estos servicios cuenta la escuela? } \\
\text { D18. Los textos escolares que usan los estudiantes en esta escuela... } \\
\text { D29. En su centro educativo, ¿ocurre que la cantidad de postulaciones } \\
\text { excede la cantidad de cupos disponibles para admitir a estudiantes? }\end{array}$ \\
\hline III. Clima & $\begin{array}{l}\text { Clima } \\
\text { escolar }\end{array}$ & 䒿 & 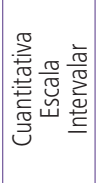 & $\begin{array}{l}\text { Cuestionario: Profesor } \\
\text { P22. En general, ¿cómo cree usted que son las relaciones dentro de la } \\
\text { escuela? } \\
\text { P23. Contamos con el apoyo del director en todo momento } \\
\text { P24. ¿Algunas de estas situaciones sucedieron en esta escuela durante el } \\
\text { último mes? }\end{array}$ \\
\hline \multirow[t]{2}{*}{$\begin{array}{l}\text { IV. } \\
\text { Gestión }\end{array}$} & Liderazgo & 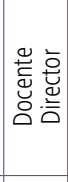 & 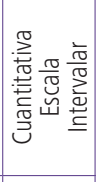 & $\begin{array}{l}\text { P26. Señale su grado de acuerdo con las siguientes afirmaciones respecto } \\
\text { a su experiencia en esta escuela } \\
\text { D25. Señale su grado de acuerdo con las siguientes afirmaciones respecto } \\
\text { a su experiencia en esta escuela } \\
\text { D28. ¿Esta escuela cuenta con un consejo escolar o instancia de gestión } \\
\text { similar? }\end{array}$ \\
\hline & $\begin{array}{l}\text { Equipo } \\
\text { directivo }\end{array}$ & 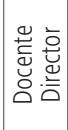 & 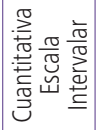 & $\begin{array}{l}\text { P27. El equipo directivo de esta escuela... } \\
\text { P28. ¿Con qué frecuencia suceden estas cosas en su escuela? } \\
\text { D24. Respecto a las decisiones sobre las siguientes acciones, ¿qué } \\
\text { participación tiene el equipo directivo u otros integrantes de la escuela? }\end{array}$ \\
\hline
\end{tabular}




\section{ANÁLISIS E INTERPRETACIÓN}

\subsection{Categorías}

El incumplimiento de la condición de unidimensionalidad significó descartar la categoría "recursos didácticos" del nivel estudiante. Los coeficientes alfa presentaron valores de consistencia aceptable a buena (entre 0,7 y 0,9) en origen social, capital cultural y entorno del aula pertenecientes al Nivel 1 y en recursos materiales, gestión y entorno del Nivel 2. Con grado suficiente (de 0,5 a 0,6 ) se obtuvo actitud hacia la escuela del Nivel 1, trabajo docente y hábitat, del Nivel 2.

TABLA 5

Estadísticos de fiabilidad de las categorías de análisis por disciplina

\begin{tabular}{lccc}
\hline \multicolumn{4}{c}{ Estadísticos de fiabilidad } \\
\hline \multicolumn{1}{c}{ Categoría } & Elementos & \multicolumn{2}{c}{ Alfa de Cronbach } \\
& 19 & 0,79945 & 0,80096 \\
\hline Origen social & 20 & 0,79432 & 0,78374 \\
Capital cultural & 15 & 0,52971 & 0,52796 \\
Actitud hacia la Escuela & 5 & 0,46347 & 0,47776 \\
Recursos Didácticos & 5 & 0,65794 & 0,67057 \\
Clima del Aula & 2 & 0,54661 & 0,54779 \\
Hábitat & 34 & 0,87230 & 0,87230 \\
Recursos Materiales & 10 & 0,54946 & 0,55177 \\
Trabajo Docente & 54 & 0,85903 & 0,85683 \\
Gestión & 15 & 0,79343 & 0,79187 \\
Clima de la Escuela & & &
\end{tabular}

En cuanto a los índices de las categorías, en lectura se obtuvo valores medios entre 0,03 y 0,34 puntos para los 1541 casos del nivel alumno y de 0,0003 a 0,96 puntos en 197 escuelas. Como desviación típica se halló de 0,28 a 2,79 puntos en el nivel 1 y entre 0,44 y 4,31 puntos para el $2^{\circ}$ nivel. Los mayores extremos se registraron en la categoría hábitat con mínimo de -7,32 y máximo de 8,65 puntos. En matemática se halló de 0,04 a 0,46 puntos medios para los 1106 casos del nivel alumno y entre 0 y 1,61 puntos para las 160 escuelas. De desviación típica se obtuvo entre 0,3 y 1,19 puntos en el nivel 1 y de 0,34 a 4,35 puntos para el $2^{\circ}$ nivel. El máximo extremo se registró en hábitat con 8,7 puntos y el mínimo de $-9,49$ en recursos materiales. 
TABLA 6

\section{Estadísticos descriptivos de las categorías de análisis por disciplina}

\begin{tabular}{|c|c|c|c|c|c|c|c|c|c|c|}
\hline \multirow[b]{3}{*}{ Categoría } & \multicolumn{10}{|c|}{ Estadísticos } \\
\hline & \multicolumn{5}{|c|}{ Lectura } & \multicolumn{5}{|c|}{ Matemática } \\
\hline & $\mathrm{n}$ & media & $\begin{array}{c}\text { desvia- } \\
\text { ción } \\
\text { típica }\end{array}$ & $\begin{array}{l}\text { míni- } \\
\text { mo }\end{array}$ & $\begin{array}{l}\text { máxi- } \\
\text { mo }\end{array}$ & $\mathrm{n}$ & $\begin{array}{c}\text { me- } \\
\text { dia }\end{array}$ & $\begin{array}{l}\text { desvia- } \\
\text { ción } \\
\text { típica }\end{array}$ & $\begin{array}{l}\text { míni- } \\
\text { mo }\end{array}$ & $\begin{array}{c}\text { máxi- } \\
\text { mo }\end{array}$ \\
\hline Origen social & 1541 & 0,34 & 1,03 & $-3,14$ & 4,38 & 1106 & 0,46 & 1,03 & $-3,30$ & 4,41 \\
\hline Capital cultural & 1541 & 0,03 & 0,33 & $-1,18$ & 2,44 & 1106 & 0,04 & 1,19 & $-4,01$ & 2,47 \\
\hline $\begin{array}{l}\text { Actitud hacia la } \\
\text { Escuela }\end{array}$ & 1541 & 0,08 & 0,28 & $-0,60$ & 1,78 & 1106 & 0,05 & 0,34 & $-1,17$ & 2,44 \\
\hline Clima del Aula & 1541 & 0,14 & 2,79 & $-6,68$ & 4,16 & 1106 & 0,09 & 0,30 & $-0,61$ & 1,82 \\
\hline Hábitat & 197 & 0,96 & 4,31 & $-7,32$ & 8,65 & 160 & 1,61 & 4,35 & $-7,34$ & 8,70 \\
\hline Recursos Materiales & 197 & $-0,0008$ & 1,31 & $-4,10$ & 3,66 & 160 & 0,36 & 2,53 & $-9,49$ & 7,93 \\
\hline Trabajo Docente & 197 & $-0,02$ &, 59 & $-1,15$ & 1,70 & 160 & 0,02 & 0,39 & $-0,88$ & 1,07 \\
\hline Gestión & 197 & $-0,0003$ &, 44 & $-1,13$ & 1,75 & 160 & 0,00 & 0,34 & $-1,89$ & 0,67 \\
\hline Clima de la Escuela & 197 & 0,653 & 2,39 & $-2,59$ & 4,98 & 160 & 0,79 & 2,41 & $-2,59$ & 4,98 \\
\hline
\end{tabular}

\subsection{Correlaciones}

El estudio enfoca las variables del nivel estudiante y su familia así como ciertas categorías del proceso de aprendizaje y gestión escolar, aplicadas al nivel escuela para relacionarlas con los resultados en las pruebas. Se constató que las categorías del Nivel 1 se relacionan linealmente en sentido directo y significativamente con las puntuaciones de las pruebas lectura y matemática.

Además, los valores del coeficiente R son mayores en lectura. Es decir, el sentido positivo indica mayor probabilidad de que valores altos de cada categoría del estudiante y su familia influyan en puntuaciones altas de las pruebas lectura y matemática. A su vez, el origen social del estudiante es la categoría de mayor relación con ambas pruebas seguido por el capital cultural. El conjunto de situaciones que los estudiantes viven en sus aulas y conforman el entorno es el tercer factor de peso.

Atendiendo la edad de los estudiantes observados cuyas familias de origen aportan un "capital inicial" es lógico encontrar más énfasis de estas relaciones en lectura. 


\section{TABLA 7}

Correlaciones de las categorías de análisis del Nivel 1 Estudiante, con los puntajes de Lectura y Matemática

\begin{tabular}{llcc}
\hline \multicolumn{1}{c}{ Categoría } & \multicolumn{1}{c}{ Estadísticos } & Lectura & Matemática \\
\hline \multirow{3}{*}{ Origen social } & Correlación de Pearson & $0,40956^{* *}$ & $0,36348^{\star *}$ \\
\cline { 2 - 4 } & Sig. (bilateral) & 0,00000 & 0,00000 \\
\cline { 2 - 4 } & $\mathrm{N}$ & 1.541 & 1.218 \\
\hline \multirow{3}{*}{ Capital cultural } & Correlación de Pearson & $0,30595^{* *}$ & $0,27031^{* *}$ \\
\cline { 2 - 4 } & Sig. (bilateral) & 0,00000 & 0,00000 \\
\cline { 2 - 4 } & $\mathrm{N}$ & 1.541 & 1.218 \\
\hline \multirow{2}{*}{$\begin{array}{l}\text { Actitud hacia la } \\
\text { Escuela }\end{array}$} & Correlación de Pearson & $0,16291^{* *}$ & $0,13739^{\star *}$ \\
\cline { 2 - 4 } & Sig. (bilateral) & 0,00000 & 0,00000 \\
\cline { 2 - 4 } Clima de Aula & $\mathrm{N}$ & 1.541 & 1.218 \\
\cline { 2 - 4 } & Correlación de Pearson & $0,21009^{* *}$ & $0,14518^{* *}$ \\
\cline { 2 - 4 } & Sig. (bilateral) & 0,00000 & 0,00000 \\
\cline { 2 - 4 } & $\mathrm{N}$ & 1.541 & 1.218 \\
\hline
\end{tabular}

**. La correlación es significativa al nivel 0,01 (bilateral).

En el Nivel escuela, las categorías hábitat y recursos materiales se relacionan significativamente en sentido directo con las puntuaciones de ambas pruebas y con valores mayores en lectura. La gestión correlaciona también linealmente en sentido positivo con la prueba de lectura pero no con la de matemática para la cual el coeficiente es negativo. Además, el entorno resultó negativo y el trabajo docente positivo pero ambos no significativos con las pruebas.

TABLA 8

Correlaciones de las categorías de análisis del Nivel 2 Escuela con los puntajes de Lectura y Matemática

\begin{tabular}{|c|c|c|c|}
\hline Categoría & Estadísticos & Lectura & Matemática \\
\hline \multirow{3}{*}{ Hábitat } & Correlación de Pearson & $0,46239 * *$ & $0,26628^{* *}$ \\
\hline & Sig. (bilateral) &, 00000 &, 00016 \\
\hline & $\mathrm{N}$ & 197 & 197 \\
\hline \multirow{3}{*}{ Recursos Materiales } & Correlación de Pearson & $0,53695^{\star *}$ & $0,41494^{* *}$ \\
\hline & Sig. (bilateral) & 00000 & 00000 \\
\hline & $\mathrm{N}$ & 197 & 198 \\
\hline \multirow{3}{*}{ Trabajo Docente } & Correlación de Pearson & 0,03904 & 0,06799 \\
\hline & Sig. (bilateral) &, 58691 & ,36180 \\
\hline & $\mathrm{N}$ & 196 & 182 \\
\hline \multirow{3}{*}{ Gestión } & Correlación de Pearson & $0,23621^{* *}$ & $-0,03384$ \\
\hline & Sig. (bilateral) &, 00089 & 63684 \\
\hline & $\mathrm{N}$ & 195 & 197 \\
\hline \multirow{3}{*}{ Clima } & Correlación de Pearson & $-0,01875$ & $-0,03071$ \\
\hline & Sig. (bilateral) & ,79323 & 66753 \\
\hline & $\mathrm{N}$ & 198 & 198 \\
\hline
\end{tabular}

**. La correlación es significativa al nivel 0,01 (bilateral). 


\subsection{Análisis Multinivel}

5.3.1 Resultados de la prueba de lectura

TABLA 9

Estimación final de los efectos fijos del modelo nulo - Lectura

\begin{tabular}{rlcccc}
\hline \multicolumn{5}{c}{ Estimación final de los efectos fijos } \\
\hline $\begin{array}{r}\text { Efecto fijo } \\
\text { intercepto 1, } \beta_{0}\end{array}$ & Coeficiente & Error típico & $t$ & g.l. aprox. & $p$ \\
intercepto 2, $\gamma_{0,0}$ & 677,9847 & 5,16497 & 131,266 & 186 & 0,000 \\
\hline
\end{tabular}

La puntuación promedio en lectura es de 677,98 para todas las escuelas y estadísticamente significativo $(p<0.001)$.

TABLA 10

Estimación final de los componentes de la varianza del modelo nulo

\begin{tabular}{lccccc}
\hline \multicolumn{5}{c}{ Estimación final de los componentes de la varianza } \\
\hline Efecto aleatorio & Desviación típica & Componentes de la varianza & g.l. & $\chi^{2}$ & $p$ \\
intercepto 1, uo & 61,54061 & 3787,24647 & 186 & 1016,03276 & 0.000 \\
nivel1, $r$ & 8,19599 & 671,74328 & & & \\
\hline
\end{tabular}

La varianza residual del nivel escuela o varianza ínter-escuelas es 3787,24647

La varianza residual del nivel estudiante o varianza intra-escuela es 671,74328

La correlación intra-clase pes 3787,24647/(3787,24647+671,74328) o sea 0,8494 Se interpreta que el $84,94 \%$ de la variabilidad de la puntuación en lectura se atribuye a las diferencias entre escuelas. Es el efecto escolar bruto y su alto valor da cuenta de la desigualdad.

TABLA 11

Componentes de la covarianza del modelo nulo

\begin{tabular}{lc}
\hline \multicolumn{2}{c}{ Componentes de la Covarianza } \\
\hline devianza & 18242,467 \\
parámetros estimados & 2 \\
valor de la función de probabilidad en la 6 iteración & $-9121,234$ \\
\hline
\end{tabular}

El modelo final se estimó con el método RML, máxima verosimilitud restringida. Para los efectos fijos, el modelo del nivel 1 contiene el intercepto $\beta_{0}$ y los índices de actitud, capital cultural, clima de aula y origen social con pendientes $\beta_{1}$ a $\beta_{4}$ e interceptos $\gamma_{1,0}$ a $\gamma_{4,0^{\prime}}$ respectivamente. Los predictores están centrados en la media del grupo y la varianza residual se ubicó en 0 . El modelo del nivel 2 contiene el 
intercepto y los índices de hábitat, recursos materiales, trabajo docente, gestión y clima de la escuela con interceptos $\gamma_{0,0}$ a $\gamma_{4,0}$. Todos los predictores están centrados en la gran media. Las ecuaciones son

Nivel 1:

$Y=\beta_{0}+\beta_{1}{ }^{*}$ Actitud ante la escuela $+\beta_{2}{ }^{*}$ Capital cultural $+\beta_{3}{ }^{*}$ Clima del aula $+\beta_{4}{ }^{*}$ Origen social+r

Nivel 2:

$\beta_{0}=\gamma_{0,0}+\gamma_{010}{ }^{*}$ hábitat $+\gamma_{0,2}{ }^{*}$ recursos materiales $+\gamma_{0,3}{ }^{*}$ trabajo docente $+\gamma_{0,4}{ }^{*}$ gestión $+\gamma_{0,5}{ }^{*}$ entorno de la escuela $+u_{0}$

Donde, $\beta_{1}=\gamma_{1,0} \quad \beta_{2}=\gamma_{2,0} \quad \beta_{3}=\gamma_{3,0} \quad \beta_{4}=\gamma_{4,0}$

TABLA 12

Estimación de los efectos fijos del modelo final de Lectura

\begin{tabular}{|c|c|c|c|c|c|c|}
\hline \multicolumn{7}{|c|}{ Estimación final de los efectos fijos } \\
\hline \multicolumn{2}{|c|}{ Efecto fijo } & Coeficiente & $\begin{array}{l}\text { Error } \\
\text { típico }\end{array}$ & $t$ & g.l. aprox. & $p$ \\
\hline \multicolumn{7}{|l|}{ intercepto $1, \beta_{0}$} \\
\hline intercepto $2, v_{0,0}$ & & 674,948 & 4.412027 & 152.979 & 181 & 0,000 \\
\hline Hábitat & intercepto $\boldsymbol{Y}_{0,1}$ & 3,992 & 1.302107 & 3.066 & 181 & 0.003 \\
\hline Recursos materiales & intercepto $\gamma_{0,2}$ & 13,897 & 3.506411 & 3.963 & 181 & 0.000 \\
\hline Trabajo docente & intercepto $\gamma_{0,3}$ & 3,469 & 3.686964 & 0.941 & 181 & 0.348 \\
\hline Gestión & intercepto $\gamma_{0,4}$ & $-9,822$ & 3.463695 & -2.836 & 181 & 0.006 \\
\hline Clima de escuela & intercepto $\boldsymbol{Y}_{0,5}$ & 0,817 & 1.847427 & 0.442 & 181 & 0.659 \\
\hline Actitud ante la escuela & $\begin{array}{l}\text { pendiente } \boldsymbol{\beta}_{1} \\
\text { intercepto } \boldsymbol{\gamma}_{1,0}\end{array}$ & $-0,164$ & 8.705287 & -0.019 & 181 & 0.985 \\
\hline Capital cultural & $\begin{array}{l}\text { pendiente } \beta_{2} \\
\text { intercepto } \boldsymbol{V}_{2,0}\end{array}$ & 22,181 & 8.331552 & 2.662 & 1531 & 0.008 \\
\hline Clima del aula & $\begin{array}{l}\text { pendiente } \beta_{3} \\
\text { intercepto } \gamma_{3,0}\end{array}$ & 4,772 & 0.889002 & 5.368 & 1531 & 0.000 \\
\hline Origen social & $\begin{array}{l}\text { pendiente } \beta_{4} \\
\text { intercepto } \boldsymbol{\gamma}_{4,0}\end{array}$ & 8,702 & 3.366119 & 2.585 & 1531 & 0.010 \\
\hline
\end{tabular}

El modelado arroja 6 (seis) factores estadísticamente significativos ( $p<0.01)$, a saber: hábitat, recursos materiales y gestión del nivel 2; origen social, capital cultural y entorno de aula del $1^{\circ}$ nivel. Cada factor aludido ejerce un efecto sobre la media de la puntuación de lectura el cual disminuyó a 674,95 puntos respecto al modelo nulo. En efecto, se tienen 3 categorías que provocarían aumentos de 22,18 puntos, de 13,897 puntos y de 8,70 puntos si, respectivamente, el capital cultural, los recursos materiales de la escuela y el origen social aumentasen 1 desviación típica. 
Con igual razonamiento, se tendrían aumentos menores en lectura, es decir, de 4,772 y 3,992 puntos si varían el entorno del aula y el hábitat de la escuela. Por el contario, el aumento de 1 desviación típica en gestión disminuiría en 9,822 puntos la prueba de lectura.

TABLA 13

Estimación de los componentes de la varianza del modelo final de Lectura

\begin{tabular}{lccccc}
\hline \multicolumn{5}{c}{ Estimación final de los componentes de la varianza } \\
\hline Efecto aleatorio & Desviación típica & Componentes de la varianza & g.l. & $\chi^{2}$ & $p$ \\
intercepto 1, u & 49,70912 & 2470,99703 & 181 & 663,49126 & 0,000 \\
nivel 1, r & 80,73568 & 6518,24978 & & & \\
\hline
\end{tabular}

La varianza del nivel escuela es menor que en el modelo nulo

TABLA 14

Componentes de la covarianza del modelo final de Lectura

\begin{tabular}{lc}
\hline \multicolumn{2}{c}{ Componentes de la Covarianza } \\
\hline Devianza & 18096,076 \\
Parámetros estimados & 2 \\
Valor de la función de probabilidad en la 6 iteración & $-9048,038$ \\
\hline
\end{tabular}

En relación al modelo nulo, la devianza es menor indicando mejor ajuste porque explica más varianza.

\subsection{Resultados de la prueba de matemática}

TABLA 15

Estimación de los efectos fijos del modelo nulo - Matemática

\begin{tabular}{rlcccc}
\hline \multicolumn{5}{c}{ Estimación final de los efectos fijos } \\
\hline $\begin{array}{r}\text { Efecto fijo } \\
\text { intercepto 1, } \beta_{0}\end{array}$ & Coeficiente & Error típico & $t$ & g.l. aprox. & $p$ \\
intercepto 2, $\boldsymbol{\gamma}_{0,0}$ & 681,4002 & 5,3259 & 127,941 & 159 & 0,000 \\
\hline
\end{tabular}

La puntuación promedio en matemática es 681,40 para todas las escuelas y estadísticamente significativo $(p<0.001)$.

TABLA 16

Estimación de los componentes de la varianza del modelo nulo - Matemática

\begin{tabular}{lccccc}
\hline \multicolumn{5}{c}{ Estimación final de los componentes de la varianza } \\
\hline Efecto aleatorio & Desviación típica & Componentes de la varianza & g.l. & $\chi^{2}$ & $p$ \\
intercepto 1, uo & 57,34405 & 3288,33994 & 159 & 775,49645 & 0,000 \\
nivel1, $r$ & 76,24505 & $5.813,30803$ & & & \\
\hline
\end{tabular}


La varianza residual del nivel escuela o varianza ínter-escuelas es 3288,33994 La varianza residual del nivel estudiante o varianza intra-escuela es 5.813,30803 La correlación intra-clase res 3288,33994/(3288,33994+5.813,30803) o sea 0,3613 El $36,13 \%$ de la variabilidad de la puntuación en matemática se atribuye a las diferencias entre escuelas. Es el efecto escolar bruto y su valor bajo indica igualdad, o sea, las escuelas aportan equitativamente a la variabilidad del puntaje.

TABLA 17

\section{Componentes de la covarianza del modelo nulo - Matemática}

\begin{tabular}{lc}
\hline \multicolumn{2}{c}{ Componentes de la Covarianza } \\
\hline Devianza & 12946,582 \\
Parámetros estimados & 2 \\
valor de la función de probabilidad en la 6 iteración & $-6473,291$ \\
\hline
\end{tabular}

El modelo final se estimó con el método RML, máxima verosimilitud restringida. Para los efectos fijos el modelo del nivel 1 contiene el intercepto $\beta_{0}$ y los índices de origen social, entorno de aula, capital cultural y actitud ante la escuela con pendientes $\beta_{1}$ a $\beta_{4}$ e interceptos $\gamma_{1,0}$ a $\gamma_{4,0^{\prime}}$ respectivamente. Los predictores están centrados en la media del grupo y la varianza residual se ubicó en 0 . El modelo del nivel 2 contiene el intercepto y los índices de hábitat, recursos materiales, trabajo docente, gestión y entorno de la escuela con interceptos $\gamma_{0,0}$ a $\gamma_{4,0}$. Todos los predictores están centrados en la gran media. Las ecuaciones de los modelos son las siguientes:

Nivel 1:

$Y=\beta_{0}+\beta_{1}{ }^{*}$ Origen social $+\beta_{2}{ }^{*}$ Clima del aula $+\beta_{3}{ }^{*}$ Capital cultural $+\beta_{4}{ }^{*}$ Actitud ante la escuela $+r$

Nivel 2:

$\beta_{0}=\gamma_{0,0}+\gamma_{0,1}{ }^{*}$ Hábitat $+\nu_{0,2}{ }^{*}$ Recursos materiales $+\nu_{0,3}{ }^{*}$ Trabajo docente $+\gamma_{0,4}{ }^{*}$ Gestión $+\nu_{0,5}{ }^{*}$ Clima de la escuela $+u_{0}$

Donde, $\beta_{1}=\gamma_{1,0} \quad \beta_{2}=\gamma_{2,0} \quad \beta_{3}=\gamma_{3,0} \quad \beta_{4}=\gamma_{4,0}$ 
TABLA 18

Estimación de los efectos fijos del modelo final de Matemática

\begin{tabular}{|c|c|c|c|c|c|c|}
\hline \multicolumn{7}{|c|}{ Estimación final de los efectos fijos } \\
\hline \multicolumn{2}{|c|}{ Efecto fijo } & Coeficiente & $\begin{array}{l}\text { Error } \\
\text { típico }\end{array}$ & $t$ & $\begin{array}{c}\text { g.l. } \\
\text { aprox. }\end{array}$ & $p$ \\
\hline \multicolumn{7}{|l|}{ intercepto $1, \beta_{0}$} \\
\hline intercepto $2, v_{0,0}$ & & 678,453 & 4,861 & 139,582 & 154 & 0,000 \\
\hline Hábitat & intercepto $\gamma_{0,1}$ & 1,143 & 1,374 & 0,832 & 154 & 0,407 \\
\hline Recursos materiales & intercepto $\gamma_{0,2}$ & 11,279 & 1,887 & 5,977 & 154 & 0,000 \\
\hline Trabajo docente & intercepto $\gamma_{0,3}$ & 3,469 & 11,537 & 0,592 & 154 & 0,554 \\
\hline Gestión & intercepto $\gamma_{0,4}$ & 2,386 & 15,099 & 0,158 & 154 & 0,875 \\
\hline Clima de escuela & intercepto $\boldsymbol{v}_{0,5}$ & 0,163 & 1,906 & 0,086 & 154 & 0,932 \\
\hline Origen social & $\begin{array}{l}\text { pendiente } \beta_{1} \\
\text { intercepto } \boldsymbol{\gamma}_{1,0}\end{array}$ & 7,622 & 4,074 & 1,871 & 1096 & 0,061 \\
\hline Clima del aula & $\begin{array}{l}\text { pendiente } \beta_{2} \\
\text { intercepto } \boldsymbol{\gamma}_{2,0}\end{array}$ & 7,733 & 2,609 & 2,963 & 1096 & 0,004 \\
\hline Capital cultural & $\begin{array}{l}\text { pendiente } \beta_{3} \\
\text { intercepto } \gamma_{3,0}\end{array}$ & 24,023 & 9,852 & 2,438 & 1096 & 0,015 \\
\hline Actitud ante la escuela & $\begin{array}{l}\text { pendiente } \beta_{4} \\
\text { intercepto } \gamma_{4,0}\end{array}$ & $-2,853$ & 8,799 & $-0,324$ & 1096 & 0,746 \\
\hline
\end{tabular}

Se obtuvo 3 (tres) factores estadísticamente significativos: recursos materiales del nivel 2; capital cultural y entorno de aula del nivel 1. La media de la puntuación de matemática bajó a 678,45 puntos respecto al modelo nulo. Con el aumento en 1 desviación típica del capital cultural, los recursos materiales de la escuela y el entorno del aula, la prueba de matemática aumentaría en 24,023 puntos; 11,279 y 7,733 puntos respectivamente.

TABLA 19

Estimación de los componentes de la varianza del modelo final de Matemática

\begin{tabular}{lccccc}
\hline \multicolumn{5}{c}{ Estimación final de los componentes de la varianza } \\
\hline Efecto aleatorio & Desviación típica & Componentes de la varianza & g.l. & $\chi^{2}$ & $p$ \\
intercepto 1, u & 47,60688 & 2266,41470 & 154 & 529,97547 & 0,000 \\
nivel 1, r & 75,34227 & 5676,45713 & & & \\
\hline
\end{tabular}

La varianza del nivel escuela es menor que en el modelo nulo.

TABLA 20

Estimación de los componentes de la covarianza del modelo final de Matemática

\begin{tabular}{lc}
\hline \multicolumn{2}{c}{ Componentes de la Covarianza } \\
\hline Devianza & 12831,718 \\
Parámetros estimados & 2 \\
Valor de la función de probabilidad en la 6 iteración & $-6415,859$ \\
\hline
\end{tabular}


En relación al modelo nulo, la devianza es menor indicando mejor ajuste porque explica más varianza.

\section{CONCLUSIONES}

El factor con mayor peso de explicación de la varianza del desempeño en lectura es el capital cultural, seguido por el origen social. De hecho, según los resultados los factores de mayor explicación del rendimiento en lectura se hallan en el Nivel 1, que corresponde a las características del estudiante. Respecto a los factores del Nivel 2 Escuela, los recursos materiales han mostrado mayor capacidad de explicación, seguido por el hábitat de los establecimientos escolares.

Cada factor ejerce un efecto sobre la media de la puntuación de lectura de modo que un cambio positivo del capital cultural, los recursos materiales de la escuela, el origen social, el entorno del aula y el hábitat de la escuela lo aumentan. Por el contario, el aumento en gestión disminuiría ${ }^{3}$ la media en la prueba de lectura. Además, es importante destacar que el $84,94 \%$ de la variabilidad de la puntuación en lectura se atribuye a las diferencias entre escuelas. Es el efecto escolar bruto y su alto valor indica desigualdad entre escuelas.

Con relación a Matemática, el 36,13\% de la variabilidad de la puntuación obtenida por los estudiantes se atribuye a las diferencias entre escuelas. Es el efecto escolar bruto y su valor bajo indica más igualdad que en el caso de lectura. El factor con mayor poder de explicación es el capital cultural, seguido por el entorno del aula, según la percepción de los estudiantes y los recursos materiales de la escuela.

Estos resultados reflejan que la posición que ocupa el estudiante en el espacio social condiciona su rendimiento. El sistema educativo paraguayo otorga una formación de mayor eficacia conforme los estudiantes aumentan sus posiciones en las categorías origen social y capital cultural (de base familiar).

Asimismo, se constata la importancia de la ubicación de la escuela, su acceso a los recursos materiales y la gestión del director como factores de la calidad educativa asociados de manera directa con el rendimiento de los estudiantes. El modelo muestra fuerte asociación entre el capital cultural del estudiante, el entorno del

\footnotetext{
${ }^{3}$ El resultado puede estar relacionado con la forma de responder de los directivos a las preguntas que integran el factor.
} 
aula y los recursos materiales de la escuela con el logro en matemática. Así como en lectura, las condiciones socioculturales son importantes y su mejoramiento retribuirá en un mayor desempeño.

Notablemente, las herramientas y estrategias pedagógicas de enseñanza y organización escolar no logran influir significativamente en la puntuación de ambas áreas, de manera tal que la escuela paraguaya puede considerarse una institución de reproducción de las condiciones sociales de origen.

De esta manera, los procesos escolares operan sobre la base de esas relaciones sociales iniciales, que son desiguales entre los tipos de familias y grupos y reafirma que la explicación de la variabilidad en el rendimiento no se concentre en la escuela. En efecto, la gestión directiva tiene un peso mucho menor con un efecto negativo exclusivamente sobre la prueba de lectura, este resultado puede estar relacionado con el enfoque administrativo de la gestión directiva, donde no se visualizan consultas sobre estrategias de acompañamiento pedagógico, mientras el trabajo docente y el entorno escolar, tampoco contribuyen a explicar el resultado de ambas pruebas.

A través de los resultados se constata que se cumplen ambas estrategias de reproducción a las que aluden Bourdieu y Passeron: las generadas por la familia y por el sistema escolar. En ese sentido, la mitad de las familias paraguayas que conformaron la muestra posee una actitud negativa hacia la escuela. Con sus estrategias educativas, docentes y directores contribuyen a reproducir las condiciones iniciales -favorables o no- esto es, la estructura del espacio social que ya opera en la dinámica de las estrategias familiares transmisoras del capital cultural. La realidad de la educación paraguaya -socialmente poco inclusiva- es la polarización entre escuelas públicas de alta concentración demográfica y bajos niveles de capital cultural respecto de las escuelas privadas, lo que trae aparejado un alto nivel de segregación.

El análisis crítico general remite a la concepción de política educativa, que actualmente descansa en las acciones realizadas por la entidad rectora, que es el Ministerio de Educación y Ciencias. Dicha política debería articular otras instancias del Estado, atendiendo que el logro escolar o la eficacia del sistema educativo es una realidad multidimensional. En ese sentido, además de lo que se disponga en el ámbito de los dispositivos pedagógicos respecto a los procesos escolares (gestión directiva y docente, currículum, estrategias de enseñanza, infraestructura), hay aspectos extraescolares que involucran a los destinatarios de la política pública, en cuanto los móviles de la desigualdad de eficacia radican en la socialización primaria 
y secundaria, en el desigual acceso a los recursos socioeconómicos, así como en la desigual dotación del capital cultural, todos factores sobre los cuales la política educativa, tal como está concebida y formulada en la actualidad, incide poco. Cabe agregar que dicha política aporta recursos insuficientes para la formación continua, el acompañamiento en aula y la profesionalización docente, elementos que mantienen el statu quo pedagógico.

\section{REFERENCIAS BIBLIOGRÁFICAS}

Baird, K.; Elías, R. (2014). Factores asociados al logro académico en Paraguay: un análisis multinivel. Revista Paraguaya de Educación, 1(4), 15-35.

Bogoya, D. (2009). Factores Asociados al Logro Académico. Caso Colombia en el SERCE. Grupo de Investigación en Evaluación de la Universidad Nacional de Colombia. Disponible en: https://sites.google.com/a/unal.edu.co/danielbogoya/3documentos-de-trabajo.

Bogoya, D., Barragán, S., Contento, M., Ocaña, A. (2014). Calibración de instrumentos de evaluación. Clasificación en matemáticas en la Universidad Jorge Tadeo Lozano. Revista Complutense de Educación, 25(2).

Bourdieu, P-F., Passeron, J-C. (1996). La Reproducción. Elementos para una teoría del sistema de enseñanza. Distribuciones Fontamara. México.

Bourdieu, P. (1997). Razones prácticas. Barcelona: Anagrama.

Bourdieu, P. (2005). Capital cultural, escuela y espacio social. México: Siglo XXI.

Cervini, R. (2002). Desigualdades en el logro académico y reproducción cultural en Argentina. Revista Mexicana de Investigación Educativa, 7(16), 445-500.

Coleman, J. S. et al. (1966). Equality of Educational Opportunity. National Center for Educational Statistics.

Fernández, T., (2004). Distribución del conocimiento escolar: clases sociales, escuelas y sistema educativo en América Latina. Tesis de Doctorado. Centro de Estudios Sociológicos. México.

Hanushek, E. A. \& Wößmann, L. (2007). Education Quality and Economic Growth. The World Bank

Hanushek, E. A. (2016). School human capital and teacher salary policies. Journal of Professional Capital and Community, 1(1), 23-40.

Herrera, S. (2010). La importancia de la educación en el desarrollo: la Teoría del Capital Humano y el Perfil Edad, Argentina. Revista Pilquen, 13. Disponible en http://www. scielo.org.ar/scielo.php?script=sci arttext\&pid=S1851-31232010000200007\&ln $\mathrm{g}=\mathrm{es} \& \ln g=\mathrm{es}$. 
Martínez-Garrido, C. (2011). 25 Investigaciones clave sobre eficacia escolar. Profesorado. Revista de currículum y formación del profesorado, XV(3).

MEC (2011). Plan Nacional de Educación 2024. Hacia el centenario de la Escuela Nueva de Ramón Indalecio Cardozo. Asunción-Paraguay.

MEC (2014). Agenda Educativa 2013-2018. Asunción-Paraguay.

Misiego, P. A, (2014) Los desafios de la docencia frente a las infancias excluidas y relegadas. Asunción del Paraguay: Instituto Desarrollo.

Murillo Torrecilla, F. (2003). Una panorámica de la investigación iberoamericana sobre eficacia escolar. REICE. Revista Electrónica Iberoamericana sobre Calidad, Eficacia y Cambio en Educación, 1(1), .Disponible en: http://www.ice.deusto.es/rinace/ reice/vol1n1/Murillo.pdf.

Ortiz, L. (2012). Educación y Desigualdad. Las clases desfavorecidas ente el Sistema educativo paraguayo. Asunción: CEADUC-Centro de Estudios Antropológicos de la Universidad Católica "Nuestra Señora de la Asunción", Asunción.

Ortiz, L. (2012). Reforma educativa y conservación social. Aspectos sociales del cambio educativo en Paraguay. Revista Latinoamericana de Estudios Educativos, XLII (4), 55-90.

Ortiz, L. (2013). Ética, trabajo y examen La formación de la disposición escolar en medios sociales desfavorecidos. Perfiles Educativos, XXXVI(143), 2014. IISUE-UNAM.

Ortiz, L. (2014). La educación escolar ante los límites de la inequidad. Debate. Revista digital de políticas públicas, 3, 4-10.

Ortiz, L. (2015). El Capital cultural a prueba. Notas provisorias sobre la desigualdad de aprendizajes. Revista Acción. Marzo de 2015. №352.

UNESCO-OREALC (2010). Factores Asociados al logro cognitivo de los Estudiantes de América Latina y el Caribe en SERCE - Segundo Estudio Regional Comparativo Explicativo. LLECE de OREALC. Santiago, Chile

UNESCO-OREALC (2014). Primera entrega de resultados del tercer estudio regional comparativo y explicativo (TERCE). Santiago, Chile

UNESCO-OREALC (2015). Informe de resultados: logros de aprendizaje. Resumen ejecutivo. OREALC/UNESCO. Santiago, Chile.

UNESCO-OREALC (2016). Reporte técnico. Tercer Estudio Reginal Comparativo y Explicativo, TERCE. Santiago, Chile. 